\title{
TDMA MAC Protocols for WiFi-based Long Distance Networks: A Survey
}

\author{
I. Hussain \\ Department of IT \\ North-Eastern Hill University \\ Shillong-793022, India
}

\author{
N. Sarma \\ Department of CSE \\ Tezpur University \\ Tezpur-784028, India
}

\author{
D. K. Saikia \\ Department of CSE \\ National Institute of Technology \\ Shillong-793003, India
}

\begin{abstract}
WiFi is widely being researched to use it beyond its original purpose of short-range communication. These kinds of efforts are stimulated due to its mass production and cost-effectiveness. WiFibased Long Distance (WiLD) networks envisage changing the rural communication scenario particularly in the undeveloped nations. However, CSMA/CA, the de-facto MAC protocol of WiFi, is found to be ill-suited for WiLD networks. As a result, various TDMA-based MAC protocols are proposed in the literature as an alternative to CSMA/CA. The proposed protocols have shown significant performance improvement in such scenarios. In this paper, we propose a classification framework of WiLD MAC protocols based on their major characteristics. Using the classification framework, a survey on the MAC protocols proposed for WiLD networks is presented. The distinctive features of the TDMA MAC protocols are critically examined by pointing out their strengths and weaknesses in WiLD environment. In addition, this paper puts forward some state of art open research challenges in this area.
\end{abstract}

\section{General Terms:}

Rural Network, MAC Layer, Wireless Communication

\section{Keywords:}

CSMA/CA, MAC Protocol, TDMA, WiFi, WiLD Network

\section{INTRODUCTION}

In recent times, WiFi has become very popular in providing Internet connectivity to the remote underserved areas using long distance links[14]. However, WiFi was originally designed to support Wireless Local Area Networks (WLANs) for short-range communication. The widespread standard for WLANs, IEEE $802.11 \mathrm{~b} / \mathrm{g}$ operating in the $2.4 \mathrm{GHz}$ frequency band, is one of the most popular wireless standards. The license-free operations in the ISM band and a variety of available low cost IEEE 802.11 hardware commodities make WiFi an attractive and economically feasible communication alternative for rural use[21]. Various research outcomes have also established the viability of WiFi as a practical solution for long distance communication.

WiLD networks are usually comprised of long distance WiFi links enabled by high-gain directional antenna covering up to several tens of kilometers[3]. A typical WiLD network architecture for rural region is explained in the following section. Many WiLD networks including research test beds are deployed in different corners of the world. Few notable real life WiLD deployments include Digital Gangetic Plains[1] in Uttar Pradesh, India, Aravind Network for Telemedicine[22] in Tamil Nadu, India, Long Distance Network in Amazonian Jungle of Peru[18] for telemedicine and telephony, and Akshaya Network[13] for e-governance in Kerala, India. Several research test beds have also been set up in recent times. MIT's Roofnet[4], QuRiNet[24] at the Quail Ridge Reserve in Napa County, California, FRACTEL[5] at IIT Bombay, India, and VillageNet[7] are some of the important WiLD network research test beds which are working towards network performance enhancement, providing support for various envisaged applications particularly real-time applications such as e-learning, e-governance, telemedicine, and telephony.

The feasibility of WiLD networks comes with a bunch of challenges. Long distance wireless links are highly unreliable due to the factors such as signal fading, and interference, which limits the overall network performance. Furthermore, the multi-hop nature of WiLD networks greatly impacts the end-to-end throughput and delay. Some of the potential objectives of WiLD network research are to further improve network performance in long distance communication, specifically to increase network throughput, reduce latency by improving spectrum usage.

Medium Access Control (MAC) protocol plays a key role in optimally utilizing the shared transmission medium which directly impacts on overall network performance. It solves the main sources of energy waste problems such as collision, idle listening, overhearing, and control packet overhead. Schedule-based protocols like Time Division Multiple Access (TDMA) provide good solutions to traffic correlation, heavy collision, contention and overhearing problems which usually occurs in contention-based channel access protocols. Many TDMA-based MAC protocols for WiLD networks have been proposed in the literature addressing issues pertaining to performance enhancement in long distance communication continuing the use of commodity 802.11 hardware. However, issues like single point of failure, interference-aware scheduling, congestion avoidance, QoS provisioning, multi-hop support, network reliability, etc., still remain unaddressed or partially addressed.

In this paper, we first define a classification framework to categorize different protocols based on factors like network control, syn- 
chronization, scheduling mechanism, slot size, etc. Then, we carry out a systematic survey on the TDMA-based MAC protocols proposed for WiLD networks. It discusses the distinctive features of the TDMA-based MAC protocols by pointing out their strengths and weaknesses. Finally, the open research issues have been listed and suggestions for possible solutions have been put forward.

The rest of the paper is organized as follows. In section 2 , the basic rural network architecture using WiLD links is described. Section 3 presents a discussion on why the standard CSMA/CA protocol is not suitable for WiLD networks rather TDMA is preferred. Section 4 presents the different criteria for classification and comparison of the TDMA-based MAC protocols for WiLD networks. An analysis and comparison of different TDMA-based MAC protocols for WiLD network is presented in section 5 The open research issues on TDMA-based MAC protocols for WiLD networks have been highlighted in section 6 The final section presents the conclusion of the paper.

\section{WIFI-BASED RURAL NETWORK ARCHITECTURE}

WiFi-based rural networks comprise of long distance point-to-point (P2P) and point-to-multipoint (P2MP) links enabled by high-gain directional antennae. Such links usually ranges from a few kms up to a few tens of kms to cover long distances in rural areas. The gateway node in WiLD network is ordinarily located in the district headquarters which are connected to high speed Internet through fiber optic or other communication media. Rural network backbone consists of multiple intermediate nodes usually formed by using high-raise towers of about 25-50 meters height. The nodes are connected by long distance P2P wireless links with high-gain (23$27 \mathrm{dBi}$ ) directional antennae and beam-width of about $8^{0}$. Bhagwat et al.[3] achieved a $38 \mathrm{kms}$ of WiLD link by setting a pair of $100 \mathrm{~mW}$ transmitters connected to $23 \mathrm{dBi}$ grid-parabolic directional antennae placed on the top of high-raise $(40 \mathrm{~m})$ towers on both the ends. The village nodes are connected to the gateway through the intermediate nodes and those village nodes in turn provide connectivity to the village access points using P2MP links. A sector antenna used in a P2MP wireless link typically has a gain of about $17-19 \mathrm{dBi}$ and a beam-width of about $30^{\circ}-90^{\circ}[5]$. Each node in the network is equipped with multiple radio interfaces which enable multi-hop transmission of traffic over such links. The end points of such networks have short distance (local access) links equipped with sector or omnidirectional antennae as end-user distribution networks.

A typical WiLD network architecture proposed by FRACTEL[5] is depicted in Figure 1 which considers a network comprising of long as well as short distance local-access links. The long distance links extend connectivity from a point of wired connectivity, called the gateway to a specific point in each village. On the other hand, sector antennae at the local gateway are used to provide connectivity to the village access points using P2MP links. The architecture was based on the assumption that most of the villages can be reached from their district headquarters by a few hops; a single hop distance being about 1-40km long[3]. The local-access links extend connectivity from this point, which is termed as the localgateway, to multiple nearby locations. Such nearby locations might include individual buildings such as schools, health centers, community centers, residential buildings, etc.

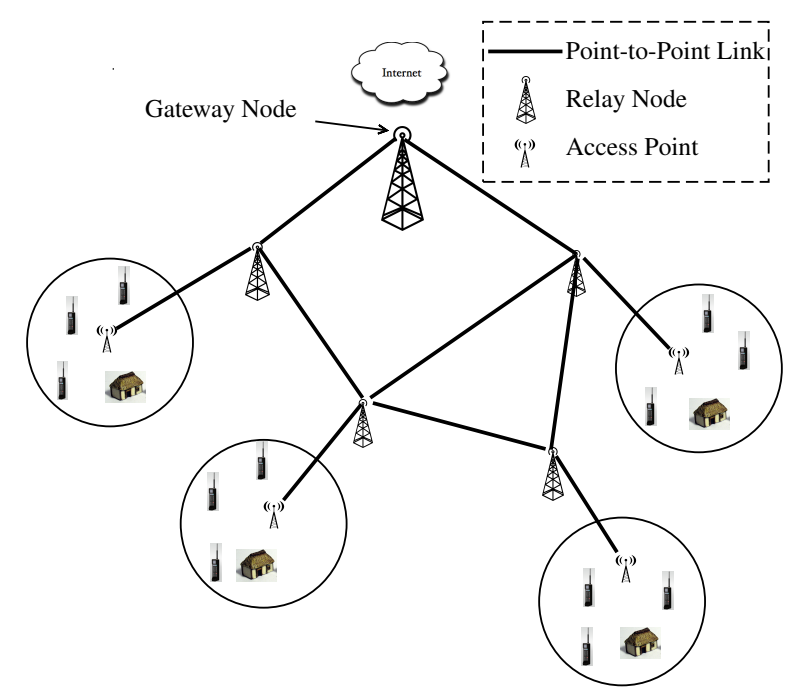

Fig. 1. WiLD Network Architecture

\section{MAC PROTOCOLS FOR WILD NETWORKS}

MAC protocols are broadly categorized into contention-based and contention-free protocols. In WiFi, CSMA/CA is the de-facto contention based MAC protocol whereas the TDMA-based MAC protocols are among the widely used contention free protocols.

CSMA/CA is a channel access mechanism which was originally designed to resolve contention in indoor conditions. In CSMA mechanism, nodes contend for the shared channel for a specified time before transmission of data, thus ensuring that the channel is free. Only after making sure that the channel is free, a node starts its transmission. If the channel is sensed busy, the node defers its transmission until it becomes idle. Collision avoidance is used to improve the performance of the CSMA method by attempting to share the channel somewhat fairly among all the transmitting nodes available within the collision domain.

TDMA channel access method is used for sharing medium among the nodes available within a common transmission range. It allows multiple stations to share the same frequency channel by dividing the transmission time into discrete slots. In TDMA mechanism, the channel is bound by a super-frame structure that consists of a number of time slots allocated by a coordinator. The consecutive time slots are separated by small period of time, called guard period. The guard period is used to ensure non-overlapping transmissions among the stations. Two stations with synchronization time less than the guard time may successfully communicate without suffering any collision. The time slots are allocated among the contending nodes according to their traffic requirements, i.e., a node gets a time slot whenever it has data to send. Since TDMA is a scheduling-based MAC scheme, nodes may turn off radios during idle times to conserve energy. Proper functioning of this MAC scheme requires all the nodes to be synchronized in time.

A comparison between CSMA and TDMA based channel access mechanisms has been provided in Table 1

TDMA utilizes available bandwidth efficiently as any number of time slots up to the maximum limit being employed can be combined to increase data throughput as required for some specific ap- 
Table 1. CSMA Vs TDMA [23]

\begin{tabular}{|c|c|c|}
\hline Performance Metrics & CSMA & TDMA \\
\hline Bandwidth Utilization & Low & Maximum \\
\hline Traffic volume Support & Low & High \\
\hline Network Scalability & Good & Poor \\
\hline Node Synchronization & Not Applicable & Required \\
\hline Power Consumption & High & Low \\
\hline
\end{tabular}

plications. Also, the volume of traffic which can be supported is high in case of TDMA since the coordinator node has absolute control over the network resources.

The CSMA/CA based networks are more scalable than TDMA. This is because of the fact that increasing the number of nodes in the network requires more slots in each frame which leads to longer transmission delay for each node. To accommodate more time slots in each frame, the length of a slot also needs to be shrunk. Although the CSMA/CA based networks are more scalable than TDMA, the overall efficiency of CSMA/CA based network goes down with the increase of traffic load. To avoid overlapping of time slots, TDMA based protocols require strict synchronization whereas CSMA-based MAC protocols do not require any established synchronization among the nodes. TDMA based MAC protocols perform better in terms of power consumption as each node may turn their radios off during other nodes time slots to save power that would otherwise be wasted contending for a busy channel.

\subsection{Performance of CSMA/CA and TDMA in WiLD Network}

While designing low cost WiLD networks by using off-the-shelf WiFi hardware, the existing CSMA/CA MAC protocol shows detrimental performance [5 11, 14]. The expected performance parameters of such networks in supporting various applications including real-time applications such as e-learning, tele-medicine, etc., cannot be delivered by the existing MAC protocols unless redesigned or tuned properly.

The standard 802.11 MAC protocol uses contention-based CSMA/CA channel access mechanism which was originally designed for short range communication. As it was not designed for long distance operation, real WiLD links show abysmal end-to-end performance [5, 11, 2]. The key reasons for this detrimental performance are highlighted by Patra et al.[14] as (1) high probability of packet loss, (2) inefficient acknowledgement mechanism, and (3) possible interference.

3.1.1 High probability of packet loss. In CSMA/CA, nodes listen to the medium before transmitting any packet check whether the channel is free or not. However, in a long distance link, a node may start transmitting a packet remaining unaware of another packet transmission from the other end. Therefore, as the signal propagation time increases in long distance links, the probability of packet loss due to collision also increases proportionally[21].

3.1.2 Inefficient acknowledgement mechanism. The 802.11 MAC uses a simple stop-and-wait protocol for acknowledging each packet independently. Upon receiving a packet successfully, the receiver node is required to send an acknowledgement (ACK) which needs to be received by the sender within a stipulated time bound known as acknowledgement timeout (ACKTimeout). If the ACK is not received before the occurrence of timeout, the sender is required to retransmit the packet again. However, the increased propagation delay with the increase of link distance makes the sender to wait for a longer time to receive the ACK packet. It decreases the channel utilization significantly. Moreover, if the time taken for the ACK to reach the sender exceeds the ACKTimeout period, the sender will retransmit unnecessarily and thus waste bandwidth. As a result, a significant decrease in channel utilization is observed with an increase in link distance[14].

3.1.3 Interference. When adjacent WiFi links operate in the same channel or in overlapping channels, interference among them is bound to occur. This may be caused as most directional antennae have sufficiently large side lobes with a gain of 4 to $8 \mathrm{dBi}$ in addition to their main lobes [3, 16]. Therefore, strength of transmitted signals from a high power radio on a node overwhelms any packet reception on other local radios 15 .

The carrier sensing MAC is not feasible for simultaneous transmission as the radios can hear each other's transmission causing one of the radios to back-off. A node equipped with multiple radios in a multi-hop network where each of these radios transmit over pointto-point long distance links to independent receivers, the above effects lead to suboptimal throughput.

On the contrary, TDMA permits several users to share a channel by dividing the time into discrete time slots. It saves the unnecessary overhead of contention for gaining access to the shared medium. Based on the TDMA schedule generated, each node gets a particular share of non-overlapping time to transmit and thus TDMA based MAC protocols are collision-free. The main task in generating a TDMA schedule is to allocate non-overlapping time slots to each station depending on the topology, packet generation rates of a node, traffic priorities, etc. TDMA-based MAC protocols enhance network performance by ensuring simultaneous transmissions without any interference. These advantages make TDMA based MAC protocols more suitable for WiLD networks.

\section{CLASSIFICATION FRAMEWORK}

The number of TDMA-based MAC protocols reported for WiLD networks are very limited in the literature. A survey in this area demands a proper classification of the TDMA-based MAC protocols into different categories. In this section, we propose a classification framework which is used to categorize different TDMA-based MAC protocols based on factors like network control, node synchronization, number of hops in the network, slot size employed, etc.

Figure 2 depicts the proposed classification framework for TDMAbased MAC protocols in WiLD environment. Different approaches of the protocols based on the classification framework have been presented. In the later part of this section, the basis of the existing MAC protocols development for WiLD networks has been provided by some traditional TDMA MAC protocols. It is then followed by an examination of the protocols and a comparative study on those.

\subsection{Classification Criteria}

4.1.1 Centralized Vs Distributed TDMA System. The centralized TDMA system has a central coordinator which takes responsibility of coordinating transmission for the whole network. This approach does not require complex distributed coordination among the nodes. But the major limitations of centralized TDMA system are: fault tolerance in presence of single node dependency, network scalability, overhead of propagating all control/contention information from/to the centralized node, and high computational overhead 


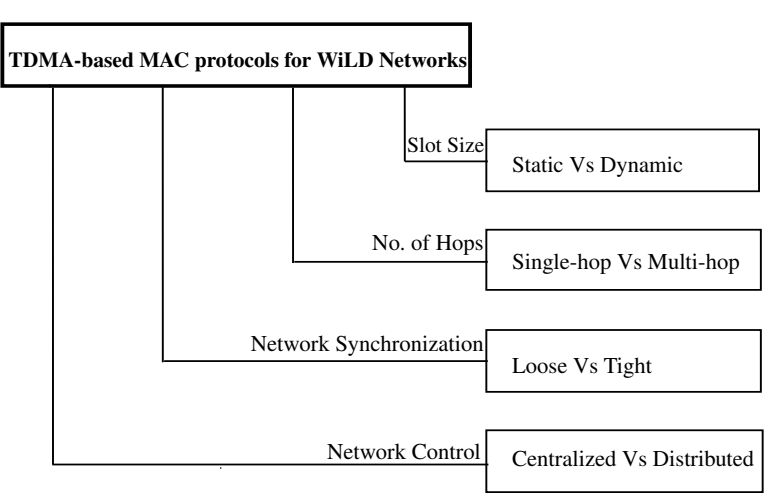

Fig. 2. Classification Framework

at the centralized node[9]. On the other hand, distributed TDMA systems provide robustness and require no global control information flow overhead. But at the same time, they require very complex implementation of the protocol mechanisms.

TDMA-based MAC protocols such as 2P, WiLDNet, and JazzyMAC rely on distributed TDMA system whereas JaldiMAC[2] and [6] use a centralized approach.

4.1.2 Tight Vs Loose Node Synchronization. In tight synchronization, all the nodes of a network are strictly synchronized to a global time or a reference time. On the other hand, in loose synchronization, the nodes are not synchronized to any global time rather they use mechanisms like token exchange.

Dhekne et al. [6] uses the power of tight synchronization to have precise control over packet transmission. Other protocols like 2P, WiLDNet, and JazzyMAC maintain loose synchronization among the nodes. JaldiMAC uses a polling based node synchronization mechanism.

4.1.3 Single-hop Vs Multi-hop Consideration. In single hop scenario, a node is only concerned about maintaining synchronization with the first hop nodes in a network. In case of multi-hop synchronization, all the nodes in the network remain synchronized irrespective of their location ( distance in number of hops from the gateway node).

Single hop synchronization is used in 2P, WiLDNet, JazzyMAC, and JaldiMAC whereas multi-hop synchronization is accomplished by Dhekne et al. in [6].

4.1.4 Static Vs Dynamic Slot Allocation. When nodes share the medium in time domain, a static slot allocation means that each node gets a constant time slot to use the shared medium. Also, the slot layout only changes when stations join or leave the network and maintain a fixed ratio between upstream and downstream traffic. But in case of dynamic slot allocation, the time slots are allocated dynamically according to the current traffic requirements.

Static slot allocation is adopted by many protocols like 2P, WiLDNet, and [6] whereas the other protocols like JaldiMAC, JazzyMAC, etc., allocate time slots among the nodes dynamically.

\section{TDMA-BASED MAC PROTOCOLS FOR WILD NETWORKS}

This section provides an overview of the existing TDMA-based MAC protocols proposed for WiLD networks. It starts with discussing some of the traditional TDMA-based MAC protocols which form the basis of the existing TDMA-based MAC protocols for WiLD networks. Later in this section, the existing TDMAbased MAC protocols for WiLD networks have been thoroughly examined and a comparative study on those protocols has been conducted.

\subsection{TDMA-based MAC Protocols}

Many TDMA-based MAC protocols for WiLD networks are proposed recently covering different scenarios such as singlehop vs. multi-hop, centralized vs. distributed, etc. However, SoftMAC[12], MadMAC[20], FreeMAC[19], and Overlay MAC Layer (OML) [17] have been found to be providing the initial platform for the development of TDMA-based MAC protocols for WiLD networks.

SoftMAC developed a software system that allows researchers to use inexpensive, commodity wireless network cards to experiment, easily construct and deploy experimental dynamic MAC layers on linux systems. MadMAC extended the idea of SoftMAC and implemented a single-hop TDMA system between two nodes with tight time synchronization. Several design challenges were addressed to maintain the persistent slot structure and continuous packet transmission. FreeMAC leveraged the methodology described in SoftMAC and implemented a single-hop TDMA system with strict timing requirements and provided a multi-channel approach.

Overlay MAC Layer (OML) is designed on the top of the 802.11 MAC layer using Click Router[10] combining the power of changing the MAC layer and the ease of modifying only the higher layers. It focuses on slot allocation to nodes according to a weighted fair queuing (WFQ) policy to improve the fairness of 802.11. OML uses loosely-synchronized clocks to divide the time into equal sized slots and then employs a distributed algorithm to allocate these slots among the competing nodes.

The TDMA-based MAC protocols discussed above are generic in nature and are not targeted for WiLD networks; however, they provide the foundation for TDMA-based MAC development for WiLD networks. 2P, WiLDNet, JazzyMAC, JaldiMAC, and [6] are some of the important TDMA-based MAC protocols which specially address the issues of WiLD MAC.

5.1.1 2P. 2P protocol[16] was the first to propose a TDMAbased approach for WiLD networks. This protocol considers the use of multi-radio operation in a single tower and demonstrates a simultaneous Synchronous Operation (SynOp) of Transmit (Tx) and Receive (Rx) in bipartite topology. It keeps a transmission link active in either of the directions all the time. The protocol uses a special synchronization packet called marker packet which acts as token. A node possessing the marker packet can only transmit for a given duration of time. When the transmission is over, the maker packet is passed from one end of a WiLD link to the other, so that at any instant of time exactly one end of the link is in transmitting and the other is in receiving mode. The authors claim to achieve a significant performance improvement over 802.11 CSMA/CA in long distance mesh networks. 
Comments: While taking scheduling decision, the protocol imposes a constraint according to which all the links at a node remain active in a given direction, either up or down, for the same amount of time. However, this may result in reduced throughput as the routing protocol running in the network layer may require the links to be active for different durations for different directions of a link. Secondly, the loss of marker packet may leave a part of the network unsynchronized for a period of time until it is resolved; which may degrade the overall performance of the network. Also, when there is no data from the IP layer, $2 \mathrm{P}$ sends dummy filler bytes instead, just to maintain synchrony. This clearly results in wastage of power. When a node is in the Rx phase, data at the node that has to be transmitted needs to be buffered till the node switches to Tx mode. This can considerably increase the end-to-end delay of real-time data across multiple hops. Thirdly, the protocol requires exchanging of marker packets with its neighbors each time it switches its phase; it further adds to the delay and hence becomes an overhead of the protocol. Lastly, 2P implementation has been experimented in a single hop scenario whereas WiLD networks are inherently multi-hop in nature. Performance of $2 \mathrm{P}$ protocol is predicted to be degrading over multi-hop topology due to lack of its support in multi-hop link scheduling.

5.1.2 WiLDNet. WiLDNet|[14] is basically built upon 2P making some additional changes in order to further improve link utilization and to make the system robust in handling packet losses. WiLDNet uses an adaptive loss recovery mechanism that uses a combination of FEC (Forward Error Correction) and bulk acknowledgment to reduce loss rate and increase end-to-end throughput. Like 2P, WiLDNet also uses static transmission time slot with loose time synchronization.

Comments: Although WiLDNet provides adaptive loss recovery mechanism to reduce loss rate and increase end-to-end throughput, it does not take delay requirements of diverse traffic scenarios into consideration. As such, no QoS issues are addressed which are very essential for real time applications. As WiLDNet is built upon $2 \mathrm{P}$ and it does not address the major issues of $2 \mathrm{P}$, the comments for $2 \mathrm{P}$ are also applicable for WiLDNet.

5.1.3 JazzyMAC. JazzyMAC[11] provisions variable length transmission slots by which each node can adapt the length of their transmission slots in accordance with their changing traffic demands. The protocol is specifically designed to allow neighbor nodes to proceed with parallel independent transmissions which contribute to enhanced throughput. The scheduling protocol does not require the topology to be bipartite, making the protocol applicable to any arbitrary topologies and each node can use purely local information for slot adaptation. JazzyMAC is claimed to achieve superior performance over $2 \mathrm{P}$ and WiLDNet.

Comments: Although JazzyMAC solves the static slot allocation problem partially, it fails to address many important issues. First, to achieve single hop synchronization, it uses tokens similar to the marker packet used by $2 \mathrm{P}$ which may introduce unnecessary delay overhead before transmission. Second, the use of dynamic time slot may not provide any specific advantage over multi-hop networks particularly in case of unidirectional flows. In such cases, the amount of traffic forwarded by the previous hop will also need equal time in the subsequent hops to forward them successfully towards the destination node. However, the use of variable length slots may provide advantages in some localized regions of the topology.
5.1.4 JaldiMAC. JaldiMAC[2] is designed to support WiLD network architecture having point-to-multipoint transmission links. It allows dynamic traffic patterns with varying symmetry ratios to adapt with the asymmetry of Internet traffic. JaldiMAC proposes a centralized ply-based packet scheduling algorithm. The algorithm broadly classifies traffic into two different categories: latency sensitive class and bandwidth class which correspond to delay and bandwidth sensitive traffic respectively. The central node first schedules the traffic belonging to latency sensitive class meeting their maximum delay bounds one after another in the gaps left by any previous schedules; and finally the remaining gaps are assigned to traffic of bandwidth class. JaldiMAC guarantees per-session fairness, provides loose QoS guarantees for latency sensitive traffic without compromising fairness, and also uses bulk acknowledgement mechanism. The protocol handles error correction to hide packet loss from overlaying TCP traffic.

JaldiMAC is implemented in two layers. The first layer defines the high-level behavior of the JaldiMAC protocol which is referred to as JaldiTDMA. This layer is responsible for tasks such as building frame headers, calculating the TDMA schedule, error control, and station addressing. The second, i.e., lower layer is responsible for configuring the chip hardware and physical layer settings as well as providing an interface for the higher layer to inject packets over the air. JaldiTDMA is implemented using the Click Modular Router in user-space, while Jaldi9k is a linux kernel module.

Comments: JaldiMAC mainly focuses on packet level traffic scheduling in point-to-multipoint WiLD links. As, JaldiTDMA is implemented using the Click Modular Router in user-space and Jaldi9k as a linux kernel module, an interface between JaldiTDMA and Jaldi9k is required which burdens the system with unnecessary overhead. For each packet transmission, a switch from user space to kernel space and vice-versa is needed which is a costly affair.

5.1.5 A Centralized TDMA MAC for Multi-hop WiLD Network. Dhekne et al.[6] demonstrates a TDMA-based MAC for multi-hop WiLD networks using off-the-shelf inexpensive hardware. It employs a centralized multi-hop node synchronization and schedule dissemination approach. The protocol uses the control slots to distribute TDMA slots from the root node to other nodes. Contention slots are used by non-root nodes to convey traffic information to the root node. Data slots are used for actual data flow across the network using data packets. A node which wants to start a new data flow, conveys its request to the root using the contention slots. The root, after getting the request, registers the flow and allocates time slot in the TDMA schedule for it. The centralized scheduler calculates the number of TDMA slots for each node as the total number slots available in the frame divided by the number of active nodes available in the network.

Comments: The TDMA scheme proposed in [6] offers limited scalability. With the increase in number of active nodes, the data slots in a TDMA frame (considering the total data slots remains constant) available for each node will decrease gradually affecting the overall network performance. Also, the protocol does not provide any priority to real-time traffic over other traffic and thus lacks in QoS provisioning.

5.1.6 A Multi-channel TDMA MAC for WiLD Networks. The MAC protocol proposed by Dutta et al. [8] provides a new channel assignment mechanism for WiLD networks. This scheme lifts the SynOp restriction [16] of 2P and enables continuous full-duplex data transfer on every link in the network. The use of multiple channels eliminates cross-link interference and thus do not require tight 
synchronization among the nodes. Considering any link in the network as made up of two direct edges, the assignment mechanism assigns non-interfering IEEE 802.11 channels in such a way that the set of channels assigned to the outgoing links is disjoined from set of channels assigned to incoming links of a node.

Comments: Although the use of multiple channels leads to fullduplex communication but the architectural consideration incurs high cost. Moreover, IEEE 802.11 has very limited number of usable channels and thus is prone to RF pollution.

\subsection{A Comparison of WiLD MAC Protocols}

After discussing the important TDMA-based MAC protocols for WiLD networks in the previous section, a comparison of the key protocols has been presented in Table 2

Majority of the available MAC protocols such as 2P, JazzyMAC, SoftMAC, FreeMAC, and [6] are implemented at the existing MAC layer whereas WiLDNet and JaldiMAC are implemented on top of the 802.11 MAC layer. A click modular router software is used to prototype a modular MAC layer by composing different click elements together. All the protocols except [8] have avoided the use of multiple channels and stuck to single channel assumption due to the limited number of usable channels in 802.11 and avoiding RF pollution problem.

JazzyMAC and JaldiMAC use the available bandwidth by taking the actual traffic needs into account while creating dynamic transmission slot layout whereas other MAC protocols use static layout that only change when stations join or leave the network. According to [6], a node wanting to start a new data flow, first conveys its request to the root. Upon receiving the request, the root node registers the flow and allocates time slots in the schedule accordingly and thus achieves dynamic transmission slot allocation.

2P, WiLDNet, and JazzyMAC employ a distributed algorithm for synchronization of nodes which is implemented by exchanging tokens. In 2P, loose synchronization between each node and its neighbors are ensured. Like 2P, JazzyMAC also maintains loose synchronization among the nodes in the network. WiLDNet uses implicit loose synchronization that relies on time stamping of each packet sent by the sender. By examining the timestamps, a smoothing function infers when a phase switch should occur. JaldiMAC uses a polling based approach where the master transmits the downstream data in chunks, rather than all at once as done in some other protocols. A control frame included with each chunk of data indicates which station may transmit next and the length of time for which it may do so. The time at which such a control frame is received thus serves to implicitly synchronize the transmitting station with the master. The protocol proposed in [6] is the only protocol which has incorporated multi-hop tight time synchronization to achieve strict and fine control over packet transmissions in the entire network. With the use of multi-channel concept, [8] eliminates the use of any strict synchronization mechanism among the nodes in the network.

Most of the protocols consider long distance point-to-point link based WiLD network architecture whereas JaldiMAC focuses on point-to-multipoint link based architecture over long distances. JazzyMAC uses dynamic slot sizing to negotiate the delaythroughput trade-off and exploits the asymmetric and time varying traffic to achieve optimized throughput and average end-toend delay. JaldiMAC guarantees per-session fairness and provides QoS guarantee for different traffic service classes which is not
Table 2. Comparison of TDMA MAC protocols for WiLD networks

\begin{tabular}{|c|c|c|c|c|c|c|}
\hline Criteria & $2 \mathrm{P}$ & $\begin{array}{c}\text { WiLD- } \\
\text { Net }\end{array}$ & $\begin{array}{c}\text { Jazzy- } \\
\text { MAC }\end{array}$ & $\begin{array}{c}\text { Jaldi- } \\
\text { MAC }\end{array}$ & {$[6]$} & {$[8]$} \\
\hline $\begin{array}{c}\text { Implemented } \\
\text { at }\end{array}$ & $\begin{array}{c}\text { MAC } \\
\text { Layer }\end{array}$ & $\begin{array}{c}\text { Click } \\
\text { Router }\end{array}$ & $\begin{array}{c}\text { MAC } \\
\text { Layer }\end{array}$ & $\begin{array}{c}\text { Click } \\
\text { Router }\end{array}$ & $\begin{array}{c}\text { MAC } \\
\text { Layer }\end{array}$ & $\begin{array}{c}\text { MAC } \\
\text { Layer }\end{array}$ \\
\hline $\begin{array}{c}\text { Single } \\
\text { Channel }\end{array}$ & Yes & Yes & Yes & Yes & Yes & $\begin{array}{c}\text { Multi } \\
\text { Chan- } \\
\text { nel }\end{array}$ \\
\hline $\begin{array}{c}\text { Dynamic } \\
\text { Transmission } \\
\text { Slot }\end{array}$ & No & No & Yes & Yes & No & N/A \\
\hline $\begin{array}{c}\text { Multi-hop } \\
\text { Time Sync. }\end{array}$ & No & No & No & No & Yes & No \\
\hline $\begin{array}{c}\text { TDMA Slot } \\
\text { Size }\end{array}$ & Static & Static & $\begin{array}{c}\text { Dyna- } \\
\text { mic }\end{array}$ & $\begin{array}{c}\text { Dyna- } \\
\text { mic }\end{array}$ & Static & N/A \\
\hline $\begin{array}{c}\text { Time Syn- } \\
\text { chronization }\end{array}$ & Loose & Loose & Loose & Loose & Tight & Loose \\
\hline $\begin{array}{c}\text { Addressing } \\
\text { QoS Issues }\end{array}$ & No & Partial & Partial & Yes & Partial & No \\
\hline
\end{tabular}

considered by any other protocol. Although the $2 \mathrm{P}$ protocol has achieved throughput performance as much as 20 times higher than CSMA/CA in long distance mesh networks, it incurs significantly high end-to-end delays in multi-hop scenario. In provisioning QoS, JaldiMAC suggests two service classes: Latency sensitive traffic class (L class) and Bulk traffic class (B class). L class attempts to minimize latency and jitter at the cost of throughput whereas B class seeks to maximize throughput with no regard for latency or jitter.

In WiLDNet's FEC implementation, jitter is minimum in the absence of error but with an increase in error, jitter also increases. WiLDNet and JaldiMAC are the only protocols which use bulk acknowledgement techniques to prevent losses at MAC layer.

The MAC protocols for WiLD network aim to optimize the overall network performance by taking the peculiar architecture of such networks into consideration. When most of the protocols employ distributed network control, [6] argues the centralized control to be better in WiLD rural networks with limited number of hops. To accommodate traffic with varying characteristics, [2] proposes dynamic adaptation of TDMA time slots in order to provision QoS.

\section{RESEARCH ISSUES IN TDMA-BASED MAC PROTOCOLS FOR WILD NETWORK}

The WiLD MAC protocols reported in the literature address many issues but still many are yet to be taken up in order to enable such networks to be a strong candidate for rural underserved areas. Some of the important research issues are discussed below.

\subsection{Support for Multi-hop TDMA Scheduling}

In providing connectivity to the village access points from the district headquarters, the WiLD network architecture is necessarily required to be multi-hop. It has been observed that most of the above TDMA-based protocols such as 2P, WiLDNet, and JazzyMAC do not consider the entire rural backbone topology at the time of scheduling. A multi-hop TDMA scheduling considering the entire WiLD network as a single unit may provide better efficiency in terms of delay and throughput. The multi-hop scheduling should necessarily be interference-aware in order to optimize network performance. Who will take the scheduling decision in a considerably stable multi-hop mesh network? Normally, a central coordinator 
e.g., the gateway node in a WiLD network does the job of multihop scheduling. However, transmission schedule can also be prepared in a distributed manner involving all the contending nodes in the decision making process.

\subsection{Leveraging Overall Network Performance}

The overall network performance can be improved with proper examination of the factors like- (1) slot reuse, (2) optimal slot scheduling, and (3) maximum link utilization. In protocols like 2P, WiLDNet, and JazzyMAC, different nodes can simultaneously transmit on the same slot by allowing non-interfering links to be active at the same time. This kind of simultaneous transmission involves only the neighbors of a particular node and hence is localized in nature. As a result, the network performance over multiple hops becomes unpredictable.

The feasibility of SynOp in WiLD networks [16] leaves a distinctive environment for slot reuse. The consideration of slot reuse greatly impacts in generating optimal transmission schedule with the interference model at hand. Hence, achieving higher degree of slot reuse in WiLD network can provide higher link utilization which will in turn provide improved network performance.

\subsection{Quality of Service (QoS) Provisioning}

The Internet traffic dynamics plays a fundamental role in the design, control, and optimization of a network. Modern Internet is composed of heterogeneous traffic differing in terms of their characteristics and QoS requirements. The existing MAC protocols other than JaldiMAC as discussed in section 5 , either partially address or do not address the QoS issues at all. Although JaldiMAC provides QoS guarantees for different service classes but it is limited to single hop. QoS provisioning in WiLD network mainly involves two broad aspects: strict end-to-end delay and end-to-end throughput.

6.3.1 Strict End-to-End Delay. Strict end-to-end delay is required to be assured for real time applications like VoIP, video conferencing, etc. In multi-hop WiLD networks, the issue of strict endto-end delay becomes important as the end-to-end delay increases with the increase in number of hops.

Protocols like 2P, WiLDNet, JazzyMAC, etc., use tokens for maintaining loose synchronization among the neighboring nodes. Under such situations, the inter-link dependence on a node can lead to unnecessary delay due to the loss of marker packets which will have additive effect over multiple hops resulting in long end-to-end delay. It is observed that the existing MAC protocols may lack in assuring strict end-to-end delay for real-time applications over multiple hops. Therefore, the provisioning of strict end-to-end delay over multiple hops still remains unaddressed. One probable solution could be to maintain tight synchronization among the nodes of a network rather than relying on token based synchronization and employ a delay-aware scheduling algorithm.

6.3.2 End-to-End Throughput. Bandwidth is a scarce resource in WiLD networks. WiLD networks are often accompanied by funneling effect which makes bandwidth a more demanding resource towards the gateway of a network. Uncontrolled bandwidth allocation can lead to congestion in the network. As such, MAC protocols for WiLD networks need to properly allocate bandwidth to different links or traffic flow over multiple hops in order to mitigate the problem of congestion. A congestion-aware MAC scheme could be very handy in this regard. Admission control schemes based on avail- ability of resources may ensure QoS guarantees for the already admitted flows in such resource-constrained networks. Unpredictable and lossy WiLD links may also impact the performance of applications like FTP which cannot tolerate high packet loss.

\subsection{Packet Level Scheduling}

To improve the overall network performance, most TDMA-based MAC protocols focus on the link level scheduling of traffic and do not deal with packet level traffic scheduling within a node. Packets of heterogeneous traffic types having different traffic requirements remain buffered in queues for longer time. When a node is assigned a time slot for transmission, the packets with higher priority need to be forwarded earlier than the others. Therefore, a fine-tuned QoSaware packet scheduling mechanism is inevitable in order to provide QoS provisioning for different types of applications including real-time.

\subsection{Multi-channel Multi-radio Consideration}

All TDMA-based MAC protocols except [8] have avoided the use of multi-channel concept and have stuck to the use of single channel. A possible reason for this is the limited number of usable channels available in IEEE 802.11 standard and to prevent RF pollution. As an intermediate node in WiLD network is equipped with multiple radios, the use of multiple channels can further enhance the network performance manifold. A proper channel assignment scheme with multi-channel multi-radio consideration can greatly improvise the performance degradation due to single channel consideration.

\subsection{Reliability Enhancement using Multiple Gateways}

The single gateway consideration in most of the WiLD networks makes the network highly unreliable. The failure of the gateway or any other node close to the gateway can leave the network fully or partially in disconnected state. Incorporating multiple gateway nodes in WiLD network architecture which are assumed to be connected with high speed Internet can provide more reliability in transmission. With the use of multiple gateway nodes, routing issues will also be opened up. In case of a path failure, any other alternative paths can be chosen to continue uninterrupted transmission. In presence of multiple gateways, the network load can be shared among all the gateways which will avoid the problem of funneling effect towards the gateway node.

\section{CONCLUSION}

As WiFi-based long distance networks continue to develop and emerge, MAC protocols are also evolving from simple TDMAbased MAC to more adaptive ones. This paper critically examines the existing TDMA-based MAC protocols for WiLD networks. The TDMA mechanism is considered to be the most efficient and reliable for long distance wireless networks in spite of its limitations such as frequent synchronization overhead and dynamic slot time assignment. Different criteria for classification of the TDMA-based MAC protocols have also been brought out in the paper. This paper further points out some of the research issues of the MAC protocols which are yet to be addressed. Solutions to these research challenges will help in designing a stable and efficient TDMA-based MAC protocol and take a step forward in making WiFi-based networks for rural underserved areas a reality. 


\section{Acknowledgment}

This work has been supported by the project entitled "QoS Provisioning in WiFi-based Long Distance Wireless Networks for Hilly Terrain Areas" (Ref No. 14 (8)/2011-CC \& BT dated: 06-JAN-12) funded by DeitY (CC \& BT), Govt. of India.

\section{REFERENCES}

[1] RuralNet (Digital Gangetic Plains: DGP) 802.11-based LowCost Networking for Rural India. http://www.cse.iitk. ac.in/users/braman/dgp.html

[2] Yahel Ben-David, Matthias Vallentin, Seth Fowler, and Eric Brewer. JaldiMAC: taking the distance further. In Proceedings of the $4^{\text {th }}$ ACM Workshop on Networked Systems for Developing Regions. ACM, 2010.

[3] Pravin Bhagwat, Bhaskaran Raman, and Dheeraj Sanghi. Turning 802.11 Inside-Out. SIGCOMM Computer Communication Review, 34(1):33-38, 2004.

[4] John Bicket, Daniel Aguayo, Sanjit Biswas, and Robert Morris. Architecture and Evaluation of an Unplanned 802.11b Mesh Network. In Proceedings of the $11^{\text {th }}$ Annual International Conference on Mobile Computing and Networking, pages 31-42. ACM, 2005.

[5] Kameswari Chebrolu and Bhaskaran Raman. FRACTEL: A Fresh Perspective on (Rural) Mesh Networks. In Proceedings of the 2007 Workshop on Networked Systems for Developing Regions. ACM, 2007.

[6] Ashutosh Dhekne, Nirav Uchat, and Bhaskaran Raman. Implementation and Evaluation of a TDMA MAC for WiFibased Rural Mesh Networks. NSDR, Oct 2009.

[7] Partha Dutta, Sharad Jaiswal, Debmalya Panigrahi, KVM Naidu, Rajeev Rastogi, and Ajay Todimala. VillageNet: A Low-cost, 802.11-based Mesh Network for Rural Regions. In the $2^{\text {nd }}$ International Conference on Communication Systems Software and Middleware, pages 1-8. IEEE, 2007.

[8] Partha Dutta, Sharad Jaiswal, Debmalya Panigrahi, and Rajeev Rastogi. A new Channel Assignment Mechanism for Rural Wireless Mesh Networks. In INFOCOM 2008: The $27^{\text {th }}$ Conference on Computer Communications. IEEE, 2008.

[9] R Kaushik. Distributed vs Centralized TDMA MAC Comparison. $\mathrm{PhD}$ thesis, Indian Institute of Technology, Bombay, 2011.

[10] Eddie Kohler, Robert Morris, Benjie Chen, John Jannotti, and M Frans Kaashoek. The Click Modular Router. ACM Transactions on Computer Systems (TOCS), 18(3):263-297, 2000.

[11] Sergiu Nedevschi, Rabin K Patra, Sonesh Surana, Sylvia Ratnasamy, Lakshminarayanan Subramanian, and Eric Brewer. An Adaptive, High Performance MAC for Long-Distance Multihop Wireless Networks. In the $14^{\text {th }}$ ACM International Conference on Mobile Computing and Networking, pages 259-270. ACM, 2008.

[12] Michael Neufeld, Jeff Fifield, Christian Doerr, Anmol Sheth, and Dirk Grunwald. SoftMAC - Flexible Wireless Research Platform. In the $4^{\text {th }}$ Workshop on Hot Topics in Networks (HotNets-IV), Nov. 2005.

[13] Joyojeet Pal, Sergiu Nedevschi, Rabin K Patra, and Eric Brewer. A Multidisciplinary Approach to Open Access Village Telecenter Initiatives: The Case of Akshaya. E-Learning, 3(3):291-316, 2006.
[14] Rabin Patra, Sergiu Nedevschi, Sonesh Surana, Anmol Sheth, Lakshminarayanan Subramanian, and Eric Brewer. WiLDNet: Design and Implementation of High Performance WiFi Based Long Distance Networks. In the $4^{\text {th }}$ USENIX Conference on Networked Systems Design and Implementation. USENIX Association, 2007.

[15] Bhaskaran Raman and Kameswari Chebrolu. Revisiting MAC Design for an 802.11-based Mesh Network. In HotNets-III, 2004.

[16] Bhaskaran Raman and Kameswari Chebrolu. Design and Evaluation of a new MAC Protocol for Long-Distance 802.11 Mesh Networks. In the $11^{\text {th }}$ Annual International Conference on Mobile Computing and Networking, pages 156-169. ACM, 2005.

[17] Ananth Rao and Ion Stoica. An Overlay MAC Layer for 802.11 Networks. In the $3^{\text {rd }}$ International Conference on Mobile systems, Applications, and Services (MobiSys), pages 135-148. ACM, 2005.

[18] Carlos Rey-Moreno, Ines Bebea-Gonzalez, Ignacio FochePerez, River Quispe-Tacas, Leopoldo Liñán Benitez, and Javier Simo-Reigadas. A Telemedicine WiFi Network Optimized for Long Distances in the Amazonian Jungle of Peru. In the $3^{\text {rd }}$ Extreme Conference on Communication: The Amazon Expedition, ExtremeCom '11, pages 9:1-9:6. ACM, 2011.

[19] Ashish Sharma and Elizabeth M. Belding. FreeMAC: Framework for Multi-Channel MAC Development on 802.11 Hardware. In the ACM Workshop on Programmable Routers for Extensible Services of Tomorrow (PRESTO), pages 69-74. ACM, 2008.

[20] Ashish Sharma, Mohit Tiwari, and Haitao Zheng. MadMAC: Building a Reconfiguration Radio Testbed using Commodity 802.11 Hardware. In $1^{\text {st }}$ IEEE Workshop on Networking Technologies for Software Defined Radio Networks, (SDR), pages 78-83, Sep. 2006.

[21] Anmol Sheth, Sergiu Nedevschi, Rabin Patra, Sonesh Surana, Eric Brewer, and Lakshminarayanan Subramanian. Packet Loss Characterization in WiFi-based Long Distance Networks. In INFOCOM: $26^{\text {th }}$ IEEE International Conference on Computer Communications, pages 312-320. IEEE, 2007.

[22] Sonesh Surana, Rabin Patra, Sergiu Nedevschi, and Eric Brewer. Deploying a Rural Wireless Telemedicine System: Experiences in Sustainability. COMPUTER, 41(6):48-56, 2008.

[23] Sana Ullah, Bin Shen, SM Riazul Islam, Pervez Khan, Shahnaz Saleem, and Kyung Sup Kwak. A Study of MAC Protocols for WBANs. Sensors, 10(1):128-145, 2009.

[24] Daniel Wu, Dhruv Gupta, and Prasant Mohapatra. QuRiNet: A Wide-area Wireless Mesh Testbed for Research and Experimental Evaluations. Ad Hoc Networks, 9(7):1221-1237, 2011. 